\title{
Preface: evidence-based practice guideline for the treatment of chronic kidney disease
}

(C) Japanese Society of Nephrology 2009

The concept of chronic kidney disease (CKD) was proposed in the United States in 2002 and quickly spread, becoming widely accepted in Japan. This concept includes broader pathological conditions and symptoms than those of the classical renal diseases. The growing number of CKD patients who advance to end-stage renal disease and need dialysis treatment is a serious concern in government health economics. Moreover, CKD patients are shown to be highly susceptible to cardiovascular disease (CVD), and their morbidity and mortality due to CVD is high. There is an increasing need for measures and treatments to be undertaken against $\mathrm{CKD}$, and the Japanese Society of Nephrology (JSN) has taken a leading role in establishing them. At the end of 2006, the Scientific Committee of JSN decided to create an evidence-based practice guideline for the treatment of CKD. A working group consisting of 35 physicians, nephrologists, and other specialists was set up, and the members undertook a number of difficult tasks that included setting out the problems to be discussed, searching through the evidence, evaluating each piece of that evidence, make abstract tables, and issuing policy statements. After more than 2 years of hard work, the "Evidence-Based Practice Guideline for the Treatment of CKD" was completed and was published in March 2009. The guideline is intended for medical personnel in general, but its primary target is nephrologists. The therapeutic objectives are CKD patients as a whole; however, we have not included dialysis patients (CKD stage 5D). We are pleased to have created this first evidence-based guideline, published by JSN. We hope that the guideline contributes to better management and treatment of CKD patients and, ultimately, to reducing the number of CKD patients.

Sei Sasaki MD

Chairman, Scientific Committee

Japanese Society of Nephrology 